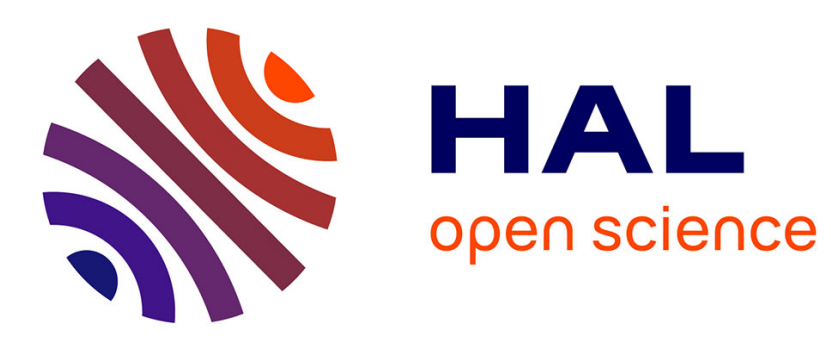

\title{
Strategies for Data Dissemination to Mobile Sinks in Wireless Sensor Networks
}

Elyes Ben Hamida, Guillaume Chelius

\section{To cite this version:}

Elyes Ben Hamida, Guillaume Chelius. Strategies for Data Dissemination to Mobile Sinks in Wireless Sensor Networks. IEEE Wireless Communications, 2008, 15 (6), pp.31-37. 10.1109/MWC.2008.4749745 . inria-00384839

\section{HAL Id: inria-00384839 \\ https://hal.inria.fr/inria-00384839}

Submitted on 15 May 2009

HAL is a multi-disciplinary open access archive for the deposit and dissemination of scientific research documents, whether they are published or not. The documents may come from teaching and research institutions in France or abroad, or from public or private research centers.
L'archive ouverte pluridisciplinaire HAL, est destinée au dépôt et à la diffusion de documents scientifiques de niveau recherche, publiés ou non, émanant des établissements d'enseignement et de recherche français ou étrangers, des laboratoires publics ou privés. 


\title{
Strategies for Data Dissemination to Mobile Sinks in Wireless Sensor Networks
}

\author{
Elyes Ben Hamida, Guillaume Chelius.
}

\begin{abstract}
A wireless sensor network (WSN) is a multihop wireless network consisting of spatially distributed autonomous sensors with sensing, computation and wireless communication capabilities. Each sensor generally has the task to monitor and measure ambient conditions, and disseminate the collected data towards a base station, or sink, for data post-analysis and processing. Many data dissemination protocols have been proposed to allow the dissemination of the collected data towards a static sink. However, mobile sinks have been shown recently to be more energy-effective than static ones. In this article existing data dissemination protocols supporting mobile sinks are summarized. Furthermore, sink mobility is analyzed as well as its impact on the energy consumption and the network lifetime.
\end{abstract}

Index Terms-Wireless multihop networks, sensor networks, mobile sinks, data dissemination/collection.

\section{INTRODUCTION}

Due to the recent technological advances in miniaturization, low-power circuit design, and efficient wireless capability, Wireless Sensor Networks (WSN) have emerged as a promising technology with numerous and various military and civil applications, such as weather monitoring, disaster management, intrusion detection, target tracking, tactical surveillance, etc. A Wireless Sensor Network is a multihop wireless network with tens, hundreds or thousands of sensors being deployed over an area of interest. Each sensor is generally a constrained device with relatively small memory resource, restricted computation capability, short range wireless transmitter-receiver and limited built-in battery. The deployment of sensors can be done in a random fashion (e.g., airplane dropping in a disaster management scenario), or placed manually in strategic locations (e.g., for intrusion detection or target tracking applications).

These sensors measure and monitor ambient conditions in the surrounding environment. Typical sensing tasks are heat, pressure, light, sound, vibration, presence of objects, etc. The measurements and monitored events are then forwarded for data post-analysis towards a more resource rich device called a base station or sink. This procedure is called data dissemination and is generally performed from the sensors generating the data, termed also source nodes, towards a static sink using a $n$ to- 1 communication paradigm, as shown on Figure 1. The sink can thus take the appropriate action according to the sensory input, e.g., sending an alarm notification or a report on the Internet or to a satellite.

Elyes Ben Hamida and Guillaume Chelius are with the INRIA ARES team, CITI Laboratory, National Institute of Applied Science of Lyon, F-69621, Villeurbanne, France. Email: elyes.ben-hamida@insa-lyon.fr, guillaume.chelius@insa-lyon.fr.

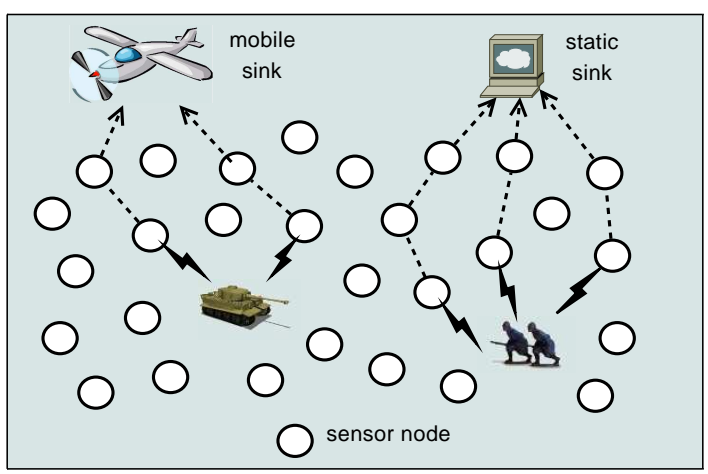

Fig. 1. Example of a Wireless Sensor Network (WSN) for target tracking and intrusion detection applications using static and mobile sinks.

Applications for wireless sensor networks fall in three major categories: (i) Periodic sensing: sensors are always monitoring the physical environment and continuously reporting measurements to the sink, such as in weather monitoring applications; (ii) Event-driven: sensors operate in a silent monitoring state and are programmed to notify about events, such as the presence of objects in intrusion detection, target tracking or military applications; and (iii) Query-based: the generated data reports are kept available within the sensor network, and sensors react to the sink's queries by returning the corresponding requested measurements and events.

Several data dissemination protocols have been proposed for wireless sensor networks with a static sink, e.g. Directed Diffusion. This approach assumes that each sink needs to periodically flood its location information through the sensor field. This procedure sets up a gradient from the sensor nodes to the sink, so that each sensor is aware of the sink location for sending future events and measurements. However, such a strategy does not scale with the network size and increases the network congestion. Moreover, the static sink may limit the network lifetime as the 1-hop neighbors of the sink are the bottleneck of the network. Exploiting mobile sinks, instead of static ones, in a wireless sensor network is thus an interesting concept to enhance the network lifetime by avoiding excessive transmission overhead at nodes that are close to the location that would be occupied by a static sink.

In such a context, the difficulty for sensor nodes is to efficiently track the mobile sink to report the collected measurements. As flat architectures and flooding-based protocols do not scale, overlaying a virtual infrastructure over the physical network has often been investigated as an efficient strategy for an effective data dissemination in presence of 
mobile sinks. This strategy is implemented in protocols like TTDD [1], GHT [2], LBDD [3], DDB [4], etc. They all use the concept of virtual infrastructure which acts as a rendez-vous area for storing and retrieving the collected measurements. The sensor nodes belonging to the rendez-vous area are designated to store the generated measurements during the absence of the sink. Once, the mobile sink crosses the network, the designated nodes are queried to report the sensory input. This virtual infrastructure can be built using a backbone-based or a rendezvous-based approach. The former is built according to a selforganizing scheme, while in the latter some localized rendezvous areas are defined within the sensor field.

In this article, we discuss the advantages of using mobile sinks and the main challenges in the design of data dissemination protocols. Next, we survey and classify the state of the art of data dissemination protocols that overlay a virtual infrastructure over the physical network to support mobile sinks. Finally, we analyze and compare, using simulations, three rendez-vous-based data dissemination protocols and we show how the considered virtual infrastructure impacts the network performance in terms of network lifetime and energy consumption.

\section{Mobile Sinks: ADVANTAGES AND DESIGN ISSUES}

\section{A. Why using a mobile sink?}

The sink mobility assumption may be useful for numerous applications. A typical application scenario is target tracking and intrusion detection. As shown on Figure 1, sensors are deployed and placed in strategic locations to monitor the battlefield area and detect enemy intrusions. When an intrusion is being detected, sensors report an alarm to the mobile sink which monitors the progression of intruders and takes the appropriate actions (e.g., sending the enemy position to the command center via a satellite). The sink represents an important component of a wireless sensor network as it acts as a gateway between sensors and the end-user.

The sink mobility assumption can be imposed by the nature of the deployed application. For example, in securityconstrained scenario the use of a mobile sink makes harder the damage of such component. Indeed, if a static sink is located, it can be easily compromised and damaged by malicious users, thus making the sensors disconnected from the enduser. On the other hand, sink mobility may improve the network connectivity by allowing the retrieval of collected measurements from several isolated parts of the sensor field. Furthermore, mobile sinks have been shown to improve the network lifetime by spreading the overhead of nodes that are close to the sink location.

\section{B. Design issues}

Despite the numerous advantages discussed above, the sink mobility brings several challenges when designing energyefficient data dissemination protocols. These issues are discussed below in more details.

Sink location and reporting method: The main goal of a sensor is to monitor the surrounding environment and to forward measurements and events towards the sink. If the sink location information is known by the sensors, data reports can be sent directly to the sink. In such a case, periodic and event-driven data reporting methods can be considered. However, in a wireless sensor network with a mobile sink, sensors do not have any a-priori knowledge of the mobile sink location. Thus, the difficulty is for sensors to efficiently track the sink and report the measurements and events. A convenient solution to this problem is to overlay a virtual infrastructure, or a backbone, over the physical network, and to exploit this structure during the data dissemination process. The underlying idea is to consider the structure as a rendezvous region for storing the generated data reports such that the mobile sink can easily collect them using a query-based data reporting method. If this virtual structure is well designed, one can achieve scalability and energy-efficiency.

Mobility support: The last issue concerns the management of the sink mobility. This can be performed using the progressive footprint chaining strategy. The sink elects among its neighbors a sink manager. This sink manager forwards the sink queries' towards the sensors, and transmits the received data reports to the sink. If the distance between the sink and its sink manager exceeds a given value, a new sink manager is chosen and a logical link towards the old sink manager is established. This approach is used by RailRoad [8], LBDD [3], DDB [4], etc.

\section{A survey on Data Dissemination Protocols}

Data dissemination protocols can be classified according to several general criteria, as shown on Table I, such as the type of the disseminated information, the target of the dissemination, and the virtual infrastructure used. More generally, virtual infrastructures can be divided into rendez-vous-based and backbone-based approaches.

\section{A. Classification of data dissemination protocols}

Data dissemination protocols can be classified according to several criteria. First, they vary in the nature of the disseminated information: (i) data dissemination: the measured data is disseminated; (ii) meta-data dissemination: a meta-data is disseminated while the measured data remains stored locally in the sensor; and (iii) sink location dissemination: the sink location is stored in the sensor field. When a node detects a new event, it determines the sink's location and the data is then forwarded to this location.

The protocols can also be classified depending on where the information is disseminated: (i) a single node: the disseminated information is stored in a particular node usually chosen in a deterministic and/or geographic way; (ii) a node out of a group of nodes: a group of nodes is defined and the information is disseminated towards one node out of this group, generally the closest to the source; and (iii) a set of nodes: the information is replicated over a set of nodes.

Finally, protocols vary in the virtual infrastructure formed by the set of potential storing nodes. In general, virtual infrastructures can be divided into rendez-vous-based approaches and backbone-based approaches. In both cases, the virtual infrastructure acts as a rendez-vous region for the queries and 


\begin{tabular}{ccccc}
\hline Protocols & $\begin{array}{c}\text { Disseminated } \\
\text { information }\end{array}$ & $\begin{array}{c}\text { Data reports } \\
\text { location }\end{array}$ & $\begin{array}{c}\text { Virtual } \\
\text { infrastructure }\end{array}$ & $\begin{array}{c}\text { Position } \\
\text { awareness }\end{array}$ \\
\hline GHT [2] & data & 1 node & hashed location & yes \\
Locators [5] & sink location & 1 out of N nodes & hashed location & yes \\
TTDD [1] & data & 1 node & grid & yes \\
QDD [6] & data & 1 out of N nodes & quad-tree & yes \\
LBDD [3] & data & 1 out of N nodes/replication & line/strip & yes \\
XY [7] & data & replication & random line & yes \\
Railroad [8] & meta-data & 1 out of N nodes & rail & yes \\
HCDD [9] & data & 1 out of N nodes & clusters & no \\
DDB [4] & data & 1 out of N nodes & backbone & no \\
\hline
\end{tabular}

TABLE I

DATA DISSEMINATION PROTOCOLS IMPLEMENTING A VIRTUAL INFRASTRUCTURE: A SUMMARY.

the generated data. This concept brings several advantages. First, a virtual infrastructure allows to gather all the generated data in the network, and permits to carry out some data optimisations (e.g., data aggregation) before sending them to the sink. Second, in scenarios where sensors are deployed in an hostile environment, source nodes can be put in jeopardy by several risk factors (e.g., wildfire, seism, etc), and thereby the risk of losing some important data is high. To make durable the generated data, the source node can disseminate the data towards the rendez-vous area instead of storing them locally. Replication mechanisms can then be used inside the virtual infrastructure to ensure data persistence against node failure or malicious nodes.

However, the concept of virtual infrastructure presents some drawbacks. A rendez-vous area can become a bottleneck in the sense that all the generated data and queries are concentrated on that area. To prevent the rendez-vous area from being a bottleneck, it is possible to design a large infrastructure to better distribute the load among the nodes, at the cost of a higher data lookup overhead. For the rest of this section we survey the state-of-the-art data dissemination protocols for the rendez-vous-based and the backbone-based approaches.

\section{B. Rendez-vous-based approaches}

The first category of data dissemination protocols with mobile sinks is the rendez-vous-based virtual infrastructure. Each node is aware of its geographic location through the use of GPS or some virtual coordinate system. This is not an utopian assumption as in most sensor applications (e.g., target tracking, intrusion detection, etc.) the data is generally strongly correlated to the geographic location. Once the sensors are deployed, a virtual infrastructure is built over the physical network such that its location is known or can be determined easily by the sensors and the sink. This overlay is then used during the data dissemination process using a geographic routing algorithm. Several protocols, implementing a rendezvous-based virtual infrastructure, have been proposed in the literature. They vary in the virtual structure formed by the set of potential storing nodes. In the rest of this subsection we summarize these protocols.

TTDD: In TTDD [1], upon the detection of a new event, the source node pro-actively builds a virtual grid structure, as shown on Figure 2(a). The source node chooses itself as the start crossing point in the grid and then sends a notification to its four adjacent crossing points of the grid. This operation continues until the grid is completely built. A sink can thus transmit a query which propagates along the grid to reach the source node. The data reports are then transmitted directly to the sink. When the target is mobile, the number of sources and grids increases and then may limit the network lifetime.

QDD: The QDD [6] protocol defines a common hierarchy of data forwarding nodes created by a Quadtree-based partitioning of the physical network into successive quadrants, as shown on Figure 2(e). In this approach, when a source node detects a new event, it calculates a set of rendez-vous points by successively partitioning the sensor field into four equally logical quadrants, and the data reports are sent to the nodes which are closer to the centroid of each successive partition. The mobile sink follows the same strategy for the query packet transmission. The main drawback of this approach is that few static nodes will be selected as rendez-vous points inducing a hot spot problem which may decrease the network lifetime and reliability.

GHT: Geographic Hash Table [2] was not designed to support mobile sink but can be easily adapted. In GHT, the data-report type is hashed into geographic coordinates, and the corresponding data reports are stored in the sensor node, called home-node, which is the closest to these coordinates. This home-node acts as a rendez-vous node for storing the generated data-reports of a given type. As shown on Figure 2(d), there are as much home-nodes as data types. The main drawback of this approach is the hot spot problem as all data reports and queries, for a same meta-data, are concentrated on the same home-node. This may restrict the scalability and the network lifetime.

Locators: In [5], the authors propose a dissemination model using geographic routing with locators that track the sink locations and reply sinks' location query from sensors. This locators are selected using a deterministic geographic hash function and replicated uniformly into the whole sensor field. When a sink moves, an update location message is sent to the closest four locators. To obtain a sink's location, a source node requests recursively sink's location from locators, and then sends its data reports directly to the sink.

RailRoad: Railroad [8] adopts a virtual infrastructure called a rail which is placed in the middle area of the network, as shown on Figure 2(b). The nodes inside this rail are called rail-nodes. When a source generates a new event, the corresponding data report is stored locally and a corresponding meta-data is forwarded to the nearest rail-nodes. Once a sink 


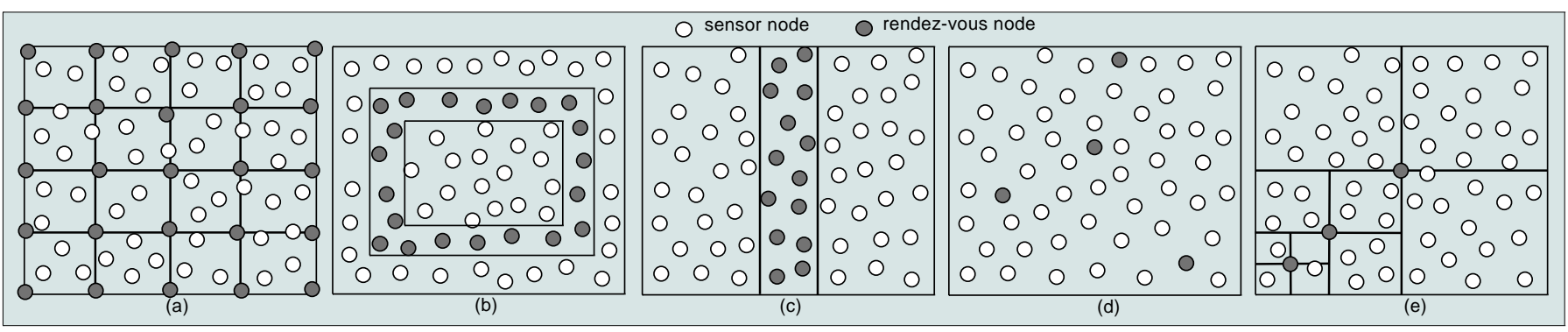

Fig. 2. Rendez-vous-based data dissemination protocols: (a) grid structure (e.g., TTDD); (b) rail structure (e.g., RailRoad); (c) line-based structure (e.g., $L B D D$ ); (d) hashed location (e.g., GHT); and (e) quad-tree structure (e.g., QDD).

node needs to collect the generated data reports, a query message is sent into the rail. This message travels around the rail until it reaches the rail-nodes which store the relevant source node information.

LBDD: LBDD [3] defines a vertical line or strip which divides the sensor field into two equal parts, as shown on Figure 2(c). Nodes within the boundaries of this wide line are called inline-nodes. This line acts as a rendez-vous area for data storage and lookup. When a sensor detects a new event, it transmits a data report towards the virtual line. This data is stored on the first inline-node encountered. To collect the generated data reports, the sink sends its query towards the rendez-vous area. The query is then propagated along the virtual line until arriving to the inline-node that owns the requested data. Data reports are thus sent directly to the sink.

XY: The Column-Row Location Service [7] is a proactive location service which can be used to disseminate data reports. In $\mathrm{XY}$, source nodes disseminate and replicate the data reports in the north and south direction, according to their current location, such that sink queries can intersect it subsequently.

\section{Backbone-based approaches}

The second category of data dissemination protocols with mobile sinks is the backbone-based virtual infrastructure. The underlying idea is to use a self-organization scheme to build a virtual structure (e.g., cluster, backbone, dominating set) over the physical network to facilitate the process of data dissemination. Such approach has already been used for routing optimization, broadcasting and topology control in wireless sensor and adhoc networks. Once the virtual infrastructure is built, data dissemination protocols can be implemented on top of this scheme with the virtual structure acting as a rendezvous area for data reports and queries. In the rest of this subsection we survey the backbone-based data dissemination protocols.

DDB: Dynamic Directed Backbone (DDB) [4] defines a data dissemination protocol on top of a self-organization scheme called LEGOS [4]. LEGOS provides and maintains a distributed and dynamic communication structure, with low message cost, where each sensor node can be either a member, a leader or a gateway, as shown on Figure 3. The leader is in charge of all its member communications, and can be linked to another leader using a gateway node, thus forming a dynamic backbone. When a sink crosses the network, it attaches itself to the backbone and sends a query to the leader. This query is then propagated along the backbone until reaching the leader owning the requested data. This procedure allows to direct the backbone such that data reports can be easily sent from leaders to the sink.

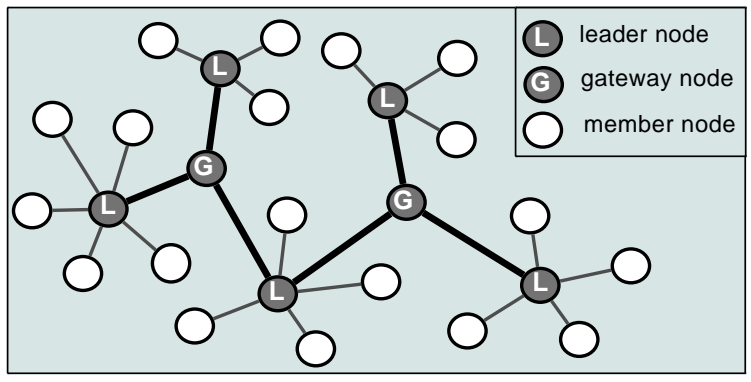

Fig. 3. Backbone-based data dissemination protocol.

HCDD: The Hierarchical Cluster-based Data Dissemination (HCDD) protocol [9] defines a hierarchical cluster architecture to keep the location of mobile sinks and find paths for the data dissemination from the sensors to the sink. Each cluster is composed by a cluster head, several gateways and ordinary sensors. The concept is similar to the one presented on Figure 3. When a mobile sink crosses the network, it registers itself to the nearest cluster head. A notification message is then propagated to all cluster heads. During this procedure, each cluster head records the sink ID and its sender such that future data reports transmission can be easily performed from sources to sink.

The main drawback of the backbone-based approach is the need to maintain the structure. In addition, the hot spot problem can occur as the traffic is concentrated over a group of cluster heads or leaders.

\section{Performance Evaluation}

As highlighted in Section III-A, the use of a virtual infrastructure for the data dissemination can lead to the hot spot problem. Indeed, as all data reports and queries are concentrated over the rendez-vous area, the hot spot problem can arise, limiting thus the network lifetime and the scalability. To prevent the rendez-vous area from being a bottleneck, it is possible to design a large virtual infrastructure to better distribute the load among its nodes. However, using a large infrastructure implies an increase of the data lookup cost, as the query has to be flooded inside the virtual infrastructure to 


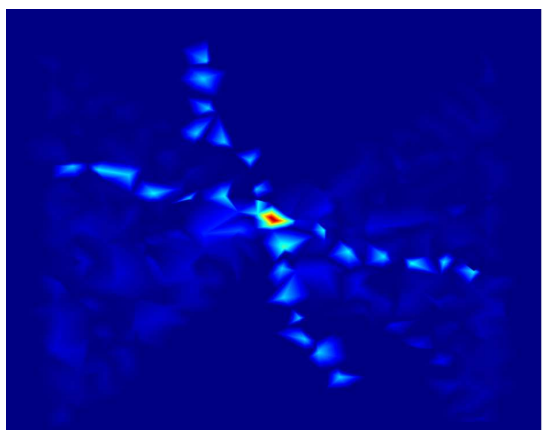

(a) GHT's energy map

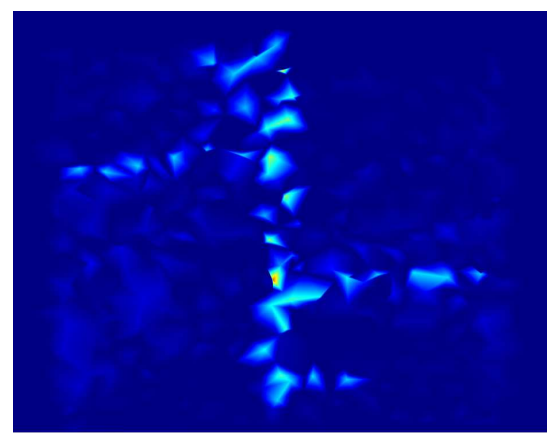

(b) LBDD's energy map

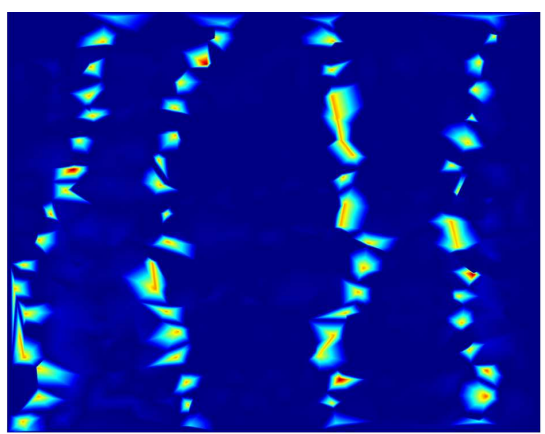

(c) XY's energy map

Fig. 4. Impact of rendez-vous-based data dissemination protocols on the energy consumption distribution after 200s of simulation.

reach the sensor node storing the requested data. There is then a clear tradeoff between the infrastructure size, the communication cost and congestion. In this section, we analyze briefly this tradeoff by mean of simulations.

\section{A. Assumptions}

As a case study, we consider the target tracking application scenario of Figure 1. A mobile target is moving within the sensor field according to an unpredictable mobility pattern. Once a sensor node detects the presence of the target (i.e., the mobile target is within reach communication of a sensor node), a message is generated and sent towards the virtual infrastructure built by the data dissemination protocol. The mobile sink can thus send its queries towards this overlay to collect the generated data. Three data dissemination protocols are considered in this comparative study: GHT [2], LBDD [3], and XY [7]. They are implemented in the WSNet simulation framework using a realistic radio medium modeling: BPSK modulation, emission power of $-25 \mathrm{dBm}$, freespace propagation model and 802.11DCF MAC protocol. Data dissemination protocols are built on top of a greedy geographic routing protocol with 1 hello packet transmission per $5 \mathrm{~s} .2000$ static nodes are deployed randomly over a $1000 \times 1000 \mathrm{~m}^{2}$ area. The sink and the target are moving according to a billiard mobility model. We assume a sink data-rate of 1 query per second and a target data-rate of 2 data reports per second. Finally, concerning energy, the radio TX consumption is assumed to be twice higher than the RX consumption. When a node energy level reaches 0 , the node silently dies and stops participating to the network. All the simulation results are averaged over 10 different runs.

\section{B. Node lifetime}

We analyze in what follows the impact of the virtual infrastructure on the nodes lifetime through the evaluation of the number of active nodes in the network during the simulations. This number decreases in function of time due to battery depletion. We plot on Figure 5-(a) the percentage of active nodes for LBDD and XY in comparison to GHT. From these results we can identify two main phases in the evolution of the network lifetime. The first phase occurs during the first $200 \mathrm{~s}$ of the simulation until the first node death in GHT. During this period, the three data dissemination protocols present the same percentage of active nodes, $100 \%$. The second phase starts from the instant $200 \mathrm{~s}$ with the sudden death of sensor nodes. The figure clearly shows an increase in the percentage of active nodes for LBDD and XY compared to GHT. Indeed, as GHT concentrates the whole traffic on a small set of rendezvous-nodes, the hot spot problem quickly occurs increasing thus the number of node premature deaths. On the other hand, the overlay of a larger rendez-vous area, as in LBDD and XY, allows to better distribute the load and the energy consumption among the sensors, and thus slowing down the sudden death of nodes.

The distribution of the energy consumption after $200 \mathrm{sec}$ onds of simulation among the sensor field is depicted on Figure 4. We observe on Figure 4-(a), that GHT presents a hot spot problem with the energy consumption being concentrated on a single rendez-vous home-node located in the square center. Concerning LBDD and XY, we notice from Figure 4(b/c) that the energy consumption is distributed among all the nodes of the rendez-vous area, which is located in the square center for LBDD (i.e., the rendez-vous area is a central strip as shown on Figure 2-(c)), and selected according to the source location for XY. This illustrates the higher percentage of active nodes obtained by LBDD and XY.

\section{Network lifetime}

We now evaluate the network lifetime. Several definitions of network lifetime can be found in the literature. In this work, we define the network lifetime from an application point of view as the time the application stops being operational which is in our case study is the time corresponding to the last report received by the sink. In other words, when the sink is no longer able to receive a report from the sensors, the sink is said to be disconnected from the sensors and the network is non-functional. To analyze the network lifetime, we plot on Figure 5-(b) the average application success ratio. This ratio is defined as the ratio between the total number of data reports received by the sink and the total number of reports generated by the sensors since the simulation beginning.

Figure 5-(b) confirms the existence of the two phases described in the previous subsection. The first phase, which we will call the regular phase, occurs up to a duration of $200 s$ and represents the normal behavior of the network when all the sensor nodes are active. During this phase, we notice 


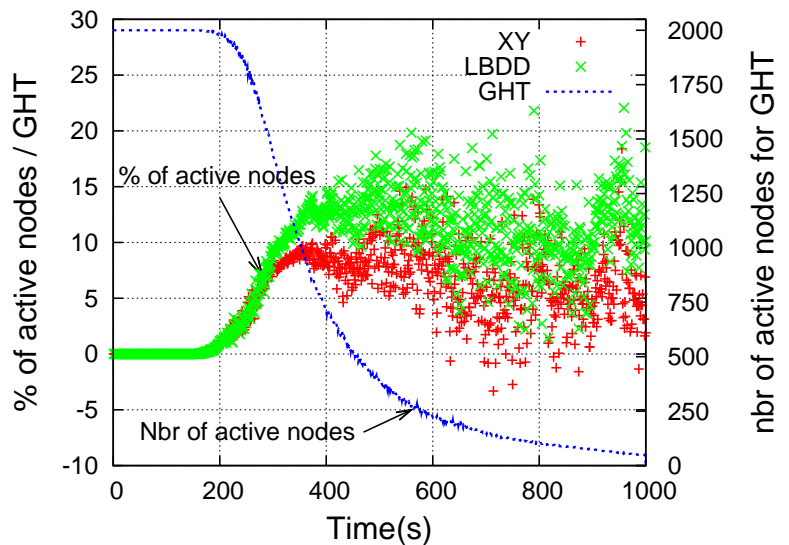

(a) Percentage of active nodes in comparison to GHT.

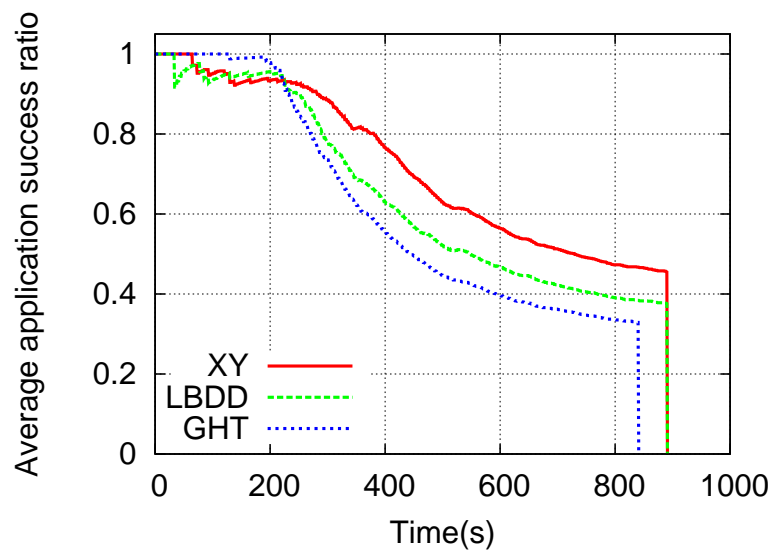

(b) Average application success ratio.

Fig. 5. Impact of rendez-vous-based data dissemination protocols on the percentage of active nodes and the average application success ratio.

that GHT presents a higher average success ratio compared to LBDD and XY. Because LBDD and XY have to flood the sink queries within the virtual infrastructure to reach the node storing the requested data, the probability of collision is thus higher and the application is less reliable. This is why their obtained average success ratio is slightly lower than GHT. Next, the second phase, the degraded phase, occurs at an instant $\approx 200 \mathrm{~s}$ and the first node deaths. During this phase, as shown on Figure 5-(a), LBDD and XY present a higher percentage of active nodes compared to GHT and a higher average success ratio. Thanks to a larger virtual infrastructure, the energy consumption of LBDD and XY is distributed over the entire rendez-vous area, avoiding thus the hot spot problem and the existence of critical nodes such as the home-nodes of GHT. It leads to a higher percentage of active nodes and introduces more redundancy between nodes, increasing the protocol robustness. This directly impacts the application success ratio which remains higher with LBDD and XY than with GHT. This confirms the analytical and experimental results of [3], [10].

\section{Conclusion}

In this article data dissemination protocols with mobile sinks are discussed. The sink mobility may be imposed by the application constraints, or it may be chosen to increase the network lifetime as well as the network connectivity. Data dissemination protocols can be classified into rendezvous-based and backbone-based approaches. They rely on the concept of a virtual infrastructure which acts as a rendezvous region for the queries and data reports. Through the study of the different approaches, we have highlighted two tradeoffs. The first one is that if, on one hand, the use of a large virtual infrastructure reduces the hot spot problem, on the other hand it increases the data lookup cost. A second tradeoff is that the use of a small virtual infrastructure may reduce the energy cost of data dissemination and collection but it may also reduce the protocol redundancy, reliability and robustness as it concentrates the traffic over a small structure, inducing congestion, premature death of nodes (i.e., overused nodes) and the existence of critical nodes.

Considering an application-oriented definition for the network lifetime has lead to the identification of two phases in the data dissemination protocol lifetime. The first one, the regular phase, corresponds to the protocol behavior before any node dies. During this phase, results show that a small virtual infrastructure offers a better performance considering data delivery. However, this phase is shorter with such an infrastructure as the first node dies earlier. During the second phase, the degraded one, the redundancy and robustness induced by a larger virtual infrastructure results in a better performance. Death of nodes occur more slowly and the protocol offers a better functioning.

These tradeoffs clearly show that the virtual infrastructure shape and the dissemination strategy must be guided by the application requirements, behavior and in particular its traffic pattern. For example, depending on the frequency of data reports and queries, a data replication over the whole virtual infrastructure may be preferable to a single-node storage. These tradeoffs also raise the question of optimal shapes that would offer a good performance during all phases of the network lifetime.

\section{REFERENCES}

[1] H. Luo, F. Ye, J. Cheng, S. Lu, and L. Zhang, "TTDD: Two-tier data dissemination in large-scale wireless sensor networks," ACM Journal of Mobile Networks and Applications (MONET), Special Issue on ACM MOBICOM (2003), 2003

[2] S. Ratnasamy, B. Karp, S. Shenker, D. Estrin, R. Govindan, L. Yin, and F. Yu, "Ght: A geographic hash table for data-centric storage in sensornets," In Proceedings of the First ACM International Workshop on Wireless Sensor Networks and Applications (WSNA'02), September 2002.

[3] E. B. Hamida and G. Chelius, "A line-based data dissemination protocol for wireless sensor networks with mobile sink," in Proceedings of the IEEE International Conference on Communications (ICC 2008), Beijing, China, May 2008.

[4] J.-L. Lu and F. Valois, "On the data dissemination in wsns," in 3rd International Conference on Wireless and Mobile Computing, Networking and Communications (WiMob), New-York, USA, October 2007.

[5] G. Shim and D. Park, "Locators of mobile sinks for wireless sensor networks," Proceedings of the 2006 International Conference on Parallel Processing Workshops (ICPPW'06), pp. 159-164, 2006.

[6] Z. H. Mir and Y.-B. Ko, "A quadtree-based data dissemination protocol for wireless sensor networks with mobile sinks," in PWC 2006, Albacete, Spain, September 2006.

[7] Y. H. S. Das, H. Pucha, "Performance comparison of scalable location services for geographic ad hoc routing," in Proceedings of IEEE INFOCOM 2005, Miami, FL, March 13-17 2005, pp. 1228-1239. 
[8] J. H. Shin, J. Kim, K. Park, and D. Park, "Railroad: virtual infrastructure for data dissemination in wireless sensor networks," Proceedings of the 2nd ACM International Workshop on Performance Evaluation of Wireless Ad Hoc, Sensor, and Ubiquitous Networks (PE-WASUN'05), pp. 168-174, Oct. 2005.

[9] C.-J. Lin, P.-L. Chou, and C.-F. Chou, "Hcdd: hierarchical clusterbased data dissemination in wireless sensor networks with mobile sink," in IWCMC '06: Proceedings of the 2006 international conference on Wireless communications and mobile computing. New York, NY, USA: ACM, 2006, pp. 1189-1194.

[10] E. B. Hamida and G. Chelius, "Analytical evaluation of virtual infrastructures for data dissemination in wireless sensor networks with mobile sink," in SANET '07: Proceedings of the First ACM workshop on Sensor and actor networks. New York, NY, USA: ACM, 2007, pp. 3-10. 